\title{
Genetic Variants Associated with the Occurrence and Progression of Adolescent Idiopathic Scoliosis: A Systematic Review Protocol
}

\section{Elizabeth A. Terhune ( $\nabla$ elizabeth.a.terhune@cuanschutz.edu )}

University of Colorado Anschutz Medical Campus: University of Colorado - Anschutz Medical Campus https://orcid.org/0000-0003-0202-6936

\section{Patricia C Heyn}

University of Colorado Anschutz Medical Campus: University of Colorado - Anschutz Medical Campus

\section{Christi R Piper}

University of Colorado Anschutz Medical Campus: University of Colorado - Anschutz Medical Campus

\section{Nancy Hadley-Miller}

University of Colorado Anschutz Medical Campus: University of Colorado - Anschutz Medical Campus

\section{Protocol}

Keywords: adolescent idiopathic scoliosis (AIS), genetic studies, genome-wide association, exome sequencing, whole genome sequencing, targeted sequencing, systematic review, variants, protocol

Posted Date: May 26th, 2021

DOl: https://doi.org/10.21203/rs.3.rs-540418/v1

License: (c) (1) This work is licensed under a Creative Commons Attribution 4.0 International License. Read Full License 


\section{Abstract}

\section{Background}

Adolescent idiopathic scoliosis (AIS) is a structural lateral spinal curvature of $\geq 10^{\circ}$ with rotation. Approximately $2-3 \%$ of children in most populations are affected with AIS, and this condition is responsible for approximately $\$ 1.1$ billion in surgical costs to the U.S. healthcare system. Although a genetic factor for AIS has been demonstrated for decades, with multiple loci identified across populations, treatment options have remained limited to bracing and surgery.

Methods

The databases MEDLINE (via PubMed), Embase, Google Scholar, and Ovid MEDLINE will be searched and limited to articles in English. We will conduct title and abstract, full-text, and data extraction screening through Covidence, followed by data transfer to a custom REDCap database. Quality assessment will be confirmed by multiple reviewers. Studies containing variant-level data (i.e. GWAS, exome sequencing) for AIS subjects and controls will be considered. Outcomes of interest will include presence/absence of AIS, scoliosis curve severity, scoliosis curve progression, and presence/absence of nucleotide-level variants. Analyses will include odds ratios and relative risk assessments, and subgroup analysis (i.e. males vs. females, age groups) may be applied. Quality assessment tools will include GRADE and Q-Genie for genetic studies.

Discussion

In this systematic review we seek to evaluate the quality of genetic evidence for AIS to better inform research efforts, to ultimately improve the quality of patient care and diagnosis.

Systematic review registration

PROSPERO registration \#CRD42021243253

\section{Introduction/background}

Adolescent idiopathic scoliosis (AIS) is the most common pediatric spinal deformity, affecting $2-3 \%$ of otherwise healthy children, with a 9:1 ratio of affected females: males for severe curvatures $(1,2)$. AIS is defined as a structural lateral spinal curvature of $\geq 10^{\circ}$, and typically manifests during the pre-adolescent period of rapid growth velocity(3-5). Radiographs of adolescents with a normal spine and with AIS, as measured by Cobb angle, are provided in Fig. 1. The high prevalence of AIS across populations, combined with potential morbidities related to functional deformity, social stigma, back pain, surgical interventions, and disease, have prompted costly school screening programs for early detection of scoliosis. The true clinical dilemma is to determine which children are at risk for AIS, and once diagnosed, which children are at risk for significant curve progression. Treatment options for scoliosis have remained stagnant for decades, and spinal fusion surgery is often advised for severe progressive curvatures with life-long 
implications. Together, the annual public health cost of pediatric screening, specialty referrals, bracing and surgery for AIS exceeds $\$ 3$ billion USD annually, not accounting for adult morbidities, including chronic back pain, pulmonary and neurological complications, and secondary surgeries, thus contributing to the estimated $\$ 849$ billion in annual costs for musculoskeletal conditions(6-9).

Decades of research into AIS families and population studies have established the strong heritability of AIS with significant sibling recurrence-risk ratio for both mild and severe curvatures $(10,11)$. While genetic heritability is high, studies of AIS families and large cohorts have consistently demonstrated significant heterogeneity indicative of the complex genetic nature of this disorder $(12,13)$. Whole exome sequencing (WES) and genome-wide association studies (GWAS) have resulted in a large number of potential predisposition genetic variants, such as those in or near $L B X 1, B N C 2$ and GPR126, which have been replicated across multiple populations. However, these variants correspond to only a modest increase in risk for AIS. Additionally, the biological roles of the most promising genetic variants in relation to AIS are unknown, thus, they have not led to a mechanistic understanding of the pathology related to this disorder.

The use of genetic variants in the clinical realm as therapeutic targets has achieved some clinical utility, particularly in relation to breast and colon cancer, and, more recently with the potential modulation of viruses, such as COVID-19 $(14,15)$. In relation to AIS, in 2010, a genetic screen of 53 single nucleotide polymorphisms (SNPs) for Caucasian children ages 9-13 years with mild scoliosis was designed to predict those most at-risk of severe curve progression (16). The genetic screen marketed as the ScoliScore (Transgenomics, Inc.) garnered mixed results upon validation, and it is unclear whether it offers any information for clinical decision making beyond a patient's natural history (17-20). Nevertheless, genetic research related to AIS has continued to expand exponentially, with large population-based genome-wide association studies (GWAS)(21-27) and whole-exome sequencing (WES) $(28-36)$ studies in both families and populations. These studies vary widely based on population, methodology, number of subjects and controls, statistical analyses, and interpretation of results $(5,37-$ 40). A critical analysis of the existing information would be of great importance, not only to assist in research efforts related to AIS genetics, but also to aid in our ability to identify children for the onset of AIS, to prioritize those children at risk for AIS progression, and to develop targeted therapeutic interventions for a personalized medicine approach to this disorder (41). This manuscript is a systematic review to assist in this effort.

Our primary objective within this systematic review is to identify specific risk single-nucleotide polymorphisms (SNPs) for AIS through methodologies that provide significant variant level data (i.e. GWAS, next-generation DNA sequencing) on the diagnosis of AIS and, if diagnosed, the susceptibility of AIS curve progression.

\section{Aims And Objectives}

Our overarching aim is to inform clinicians and researchers of the current state of Idiopathic Scoliosis (IS) genetic data to better inform future research efforts, with the ultimate goal of helping to discover 
clinically actionable targets. We plan to follow a critical appraisal approach to accomplish each of the following aims:

1. To evaluate, summarize, and synthesize literature on the genetics of late-onset IS, in order to provide recommendations for further genetic studies or further functional work, with emphasis on the development of potential diagnostics and prognostics;

2. To determine the quality of current literature on IS genetics, by GRADE(42-44) and Q-Genie(45) evaluation, including the level of evidence;

3. To summarize overall study information (i.e. publication country of origin, ethnicities of study populations, area of PI expertise) from the current body of literature of IS genetics.

By doing this work, we expect to inform the field of the most well-replicated and robust genetic variants linked to IS to-date. We expect to summarize overall study information to provide a snapshot of the field of IS genetics, and in the process, we expect to identify high-priority research areas where we have identified significant gaps in the present literature. We plan to identify pros and cons of specific methodologies and provide guidance for future studies. Lastly, we expect to provide relevant information for future prioritization of basic studies as well as translational research, in the hopes of developing future diagnostics and prognostics.

\section{Methods/ Design}

This systematic review protocol was designed in accordance with the Preferred Reporting Items of Systematic Reviews and Meta-Analysis for Protocols (PRISMA-P) v2015 checklist (Supplemental File 1). The research group will follow current best practices from Cochrane guidance and recommendations for systematic reviews (46). We will follow the standards for best practices for transparent, reproducible, and ethical reporting of systematic review guided by the Preferred Reporting Items for Systematic Reviews and Meta-Analyses Protocols (PRISMA-P) Statement(47-49). An overview of the methodology for this systematic review are provided in Fig. 2.

\section{Eligibility criteria}

The protocol has been registered in PROSPERO (50) (CRD42021243253). Included studies had to focus on idiopathic adolescent or "late-onset" scoliosis and genetic testing. Studies to be included must report data at the nucleotide level, such as genome-wide association studies, targeted sequencing, whole genome sequencing, and whole exome sequencing. Studies will be excluded if they report linkage or other data not at the nucleotide level, transcriptomic and proteomic data, or contain incomplete datasets. Conference abstracts, editorials, and reviews will also be excluded. Included studies will be limited to English language publications.

\section{Information Sources}


A comprehensive literature search will be designed and performed by a medical librarian (CP) for the concepts of: idiopathic adolescent scoliosis and genetic testing. Relevant publications will be identified by searching the following databases with a combination of standardized index terms and keywords: Ovid MEDLINE ALL (1946 to present), Embase (via Elsevier, 1947 to present), MEDLINE (via Pubmed.gov), Web of Science Core Collection (via Thomson Reuters, including Science Citation Index Expanded 1974 to present, and Social Sciences Citation Index 1974 to present), and Google Scholar (where Publish or Perish software (51) allowed downloading of the first 200 search results). Searches will be limited to English language studies. The preliminary search strategies were conducted in April 2021 and identified 1297 records. The search strategies were peer reviewed by an external librarian using the Peer Review of Electronic Search Strategies checklist(52). All results will be exported to and deduplicated in EndNote 20 (Clarivate). Covidence systematic review software (Veritas Health Innovation) will be used for screening and full text review. See Supplemental File 2 for a list of all database search strategies.

\section{Study Selection}

Citations and abstracts will be uploaded in Covidence for study selection. The study selection process is organized into two levels. For level one screening, two authors (ET, PH, and/or NHM) will independently screen all titles and abstracts. Data will be compiled at which time consensus will be reached by discussion on any disagreements for exclusion. For level two screening, full text articles considered for inclusion will be independently reviewed by two authors. Consensus will be reached by discussion on any disagreements for inclusion.

\section{Inclusion and Exclusion Criteria}

Full inclusion and exclusion criteria are provided in Supplemental File 3. In brief, inclusion criteria for studies will encompass genetic studies providing implicating specific variants (i.e. GWAS, targeted sequencing, whole genome sequencing, whole exome sequencing). Exclusion criteria includes linkage studies and other genetic studies that do not provide variant-level resolution.

\section{Data Extraction and Synthesis}

The research group will first create an Excel spreadsheet to collect relevant information from the genetic studies, followed by the creation of a REDCap database (securely managed by the University of Colorado) after the most relevant study metrics have been determined. Key data for extraction will include, but not be limited to: Number of case and control individuals, study inclusion and exclusion criteria, basic clinical and demographic information of cases and controls, genetic methodology used, statistical methods used, significant genetic variants identified and associated p-values, number of individuals in case and control groups with risk variants identified in study. Study team will select the first round of studies to be extracted with the aid of the medical librarian. Team members will review and approve all studies to be included and excluded in the SR, and will ensure that all methods are transparent, unbiased, and complete. Study team will meet weekly during the duration of the study.

\section{Quality Assessment}




\section{GRADE}

The proposed review will use the Grading of Recommendations Assessment, Development and Evaluation (GRADE) guidelines to determine the quality and strength of recommendations (42-44). Quality will be adjudicated as high (further research is very unlikely to change our confidence in the estimate of effect), moderate (further research is likely to have an important impact on our confidence in the estimate of effect and may change the estimate), low (further research is very likely to have an important impact on our confidence in the estimate of effect and is likely to change the estimate), or very low (very uncertain about the estimate of effect).

Q-Genie: We will also evaluate genetic association study quality using Q-Genie, an evaluation tool to rank genetic studies on 11 criteria based on previous study recommendations (PMID: 25975208). This tool includes ranking studies as 'low', 'moderate' or 'high' quality and assists in the selection of studies for inclusion.

\section{Expected Results}

We expect a significant degree of heterogeneity across the genetic and statistical methodologies used, sample and control groups, thresholds of significance, results, and interpretation of results across studies. We expect several findings, particularly those that have been well-replicated across multiple studies and populations, to be regarded as high quality by our evaluation.

We expect the results generated by GWAS and next-generation or targeted sequencing methodologies to produce largely different results. GWAS tends to find SNPs that are common within the general population (have a high minor allele frequency), whereas next-generation sequencing methodologies usually apply bioinformatic filters that contain only rare or uncommon variants (53). Additionally, some next-generation sequencing methodologies only look at certain areas of the genome (i.e exome sequencing analyzes coding regions only). Thus, we expect different sequencing methodologies to produce a different collection of causal variants for IS.

We also expect a high degree of overall genetic heterogeneity for IS, with the possibility of specific SNPs showing association with specific ethnic populations or subtypes of IS (38). Overall, we expect studies to show a significant genetic contribution to IS etiology $(5,38,39,54-60)$.

Lastly, we expect certain study populations to be well-represented in the literature of the genetics of IS, while we expect other ethnic groups (i.e. populations of African descent) to be understudied. Identifying understudied populations and other gaps in the current literature will assist us in making recommendations for areas of high-priority research. We anticipate that the outcomes of this systemic review will indicate that several variants implicated by GWAS and replicated across multiple ethnic groups, including $L B X 1(22,23,26,27,31,61-73)$ and $\operatorname{GPR} 126(24,27,73-78)$, will statistically associate with an increased risk of AIS development. Furthermore, we anticipate that rare or uncommon variants within extracellular matrix genes $(29,32,34,79-81)$ will collectively increase risk of AIS. Once the 
systematic review is complete, results will be disseminated through both scientific, peer-reviewed journal article(s) and national conference presentation. Any amendments made to this protocol when conducting the study will be amended in PROSPERO and reported in the final manuscript.

\section{Discussion}

This review builds upon current literature to critically assess genetic variants associated with IS risk. A recent systematic review, using eight studies from 1950-2017 that met inclusion criteria, found moderate evidence that did not clarify a single-gene basis of IS(82) (Maqsood et al., 2020). The mixed results of their study led the authors to recommend that IS researchers consider etiological factors beyond genetics alone. Three of the eight included studies supported a single-gene hypothesis for AIS etiology, albeit within specific populations. Additional systematic reviews of AIS have evaluated the quality of evidence between AIS etiology and specific variants, including those in or near $L B X 1(64-66,69,72,83), E S R 1 / 2$ (84-86), and $\operatorname{VDR}(87,88)$. Our systematic review will build upon this work by evaluating evidence for specific SNPs in relationship to AIS etiology, rather than collective evidence for a single-gene hypothesis. Based on our preliminary search strategies, we also expect to evaluate a much larger pool of research articles (> 500 articles vs 36 articles(82)).

\section{Potential challenges}

We anticipate several potential challenges with this study. First, as we are including several sequencing methodologies in this study, we anticipate a lack of common data elements across studies that may prove challenging for extraction. Second, we anticipate that some included studies will have missing data. IS is a common disorder and, without proper verification by $\mathrm{x}$-ray or physical exam, individuals with mild scoliosis can be mistakenly counted as controls.

\section{Potential limitations}

Potential limitations of this study include a lack of thorough reporting within studies (for example, inclusion/exclusion criteria, bioinformatic and statistical analyses, control databases used, lack of appropriate matching of cases and controls). A second limitation is an underrepresentation of nonCaucasian study subjects within sample populations. We also anticipate a potential lack of methodological rigor in included studies, particularly inappropriate biostatistical methodologies or inappropriate sample populations.

\section{Study implications}

This study will assist to inform best practices for future genetic studies of IS and help researchers to prioritize specific genetic loci that may warrant further research. Additionally, this study will provide a foundation for the creation of clinical genetic diagnostics to help inform a child's risk of IS development or severe curvature progression, a matter of great importance in pediatric orthopedics. 


\section{Abbreviations}

- AIS: Adolescent Idiopathic Scoliosis

- GWAS: Genome-Wide Association Study

- WES: Whole Exome Sequencing

- WGS: Whole Genome Sequencing

\section{Declarations}

Ethics approval: Not applicable.

Consent for publication: Informed consent was obtained from all individuals whose data is in this protocol.

Availability of data and materials: The data used for this study will be extracted from publications within the search databases, including PubMed, GoogleScholar, and Ovid Medline, as outlined in the Methods section.

Competing interests: NHM declares the following interests: grant funding from the National Institutes of Health, National Institutes of Arthritis and Musculoskeletal and Skin Diseases; Pediatric Orthopedic Society of North America, and Scoliosis Research Society. All other authors declare that they have no competing interests.

Data availability statement: Summary data will be provided upon publication of this systemic review. Any remaining data generated from this study will be available from the corresponding author to scoliosis researchers upon reasonable request.

Funding: Funding is provided by the Tai Foundation (Pediatric Orthopedics Section, Children's Hospital Colorado Evidence-Based Medicine Program) with additional resources from the University of Colorado Anschutz Department of Orthopedics. REDCap: This project was supported by NIH/NCATS Colorado CTSA Grant Number UL1 TR002535. Its contents are the authors' sole responsibility and do not necessarily represent official $\mathrm{NIH}$ views.

Authors' contributions: Initial preparation of manuscript (ET, CP, PH), editing of manuscript (PH, NHM), funding and resources (NHM), experimental guidance and oversight (PH, NHM)

\section{Acknowledgements}

Study data will be collected and managed using REDCap electronic data capture tools hosted at the University of Colorado Anschutz Medical Campus(89). REDCap (Research Electronic Data Capture) is a secure, web-based application designed to support data capture for research studies, providing: 1) an intuitive interface for validated data entry; 2 ) audit trails for tracking data manipulation and export 
procedures; 3 ) automated export procedures for seamless data downloads to common statistical packages; and 4) procedures for importing data from external sources.

\section{References}

1. Asher MA, Burton DC. Adolescent idiopathic scoliosis: natural history and long term treatment effects. Scoliosis. 2006;1(1):2.

2. Roach JW. Adolescent idiopathic scoliosis. Orthop Clin North Am. 1999;30(3):353-65, vii-viii.

3. Bunnell WP. The natural history of idiopathic scoliosis before skeletal maturity. Spine (Phila Pa 1976). 1986;11(8):773-6.

4. Bunnell WP. The natural history of idiopathic scoliosis. Clinical orthopaedics and related research. 1988(229):20-5.

5. Burwell RG. Aetiology of idiopathic scoliosis: current concepts. Pediatr Rehabil. 2003;6(3-4):137-70.

6. Bozzio AE, Hu X, Lieberman IH. Cost and Clinical Outcome of Adolescent Idiopathic Scoliosis Surgeries-Experience From a Nonprofit Community Hospital. Int J Spine Surg. 2019;13(5):474-8.

7. Yawn BP, Yawn RA. The estimated cost of school scoliosis screening. Spine (Phila Pa 1976). 2000;25(18):2387-91.

8. United States Bone and Joint Initiative: The Burden of Musculoskeletal Diseases in the United States (BMUS), Third Edition Rosemont, IL2014 [Available from: http://www.boneandjointburden.org.

9. United States Bone and Joint Initiative: The Burden of Musculoskeletal Diseases in the United States (BMUS), Fourth Edition Rosemont, IL2020 [Available from: http://www.boneandjointburden.org.

10. Ward K, Ogilvie J, Argyle V, Nelson L, Meade M, Braun J, et al. Polygenic inheritance of adolescent idiopathic scoliosis: a study of extended families in Utah. Am J Med Genet A. 2010;152A(5):1178-88.

11. Tang NL, Yeung HY, Hung VW, Di Liao C, Lam TP, Yeung HM, et al. Genetic epidemiology and heritability of AIS: A study of 415 Chinese female patients. J Orthop Res. 2012;30(9):1464-9.

12. Grauers A, Danielsson A, Karlsson M, Ohlin A, Gerdhem P. Family history and its association to curve size and treatment in 1,463 patients with idiopathic scoliosis. European spine journal : official publication of the European Spine Society, the European Spinal Deformity Society, and the European Section of the Cervical Spine Research Society. 2013;22(11):2421-6.

13. Inoue M, Minami S, Kitahara H, Otsuka Y, Nakata Y, Takaso M, et al. Idiopathic scoliosis in twins studied by DNA fingerprinting: the incidence and type of scoliosis. J Bone Joint Surg Br. 1998;80(2):212-7.

14. Lima ZS, Ghadamzadeh M, Arashloo FT, Amjad G, Ebadi MR, Younesi L. Recent advances of therapeutic targets based on the molecular signature in breast cancer: genetic mutations and implications for current treatment paradigms. J Hematol Oncol. 2019;12(1):38.

15. Horowitz JE, Kosmicki JA, Damask A, Sharma D, Roberts GHL, Justice AE, et al. Common genetic variants identify targets for COVID-19 and individuals at high risk of severe disease. medRxiv. 2021. 
16. Carlson B. ScoliScore AIS Prognostic Test Personalizes Treatment for Children With Spinal Curve. Biotechnol Healthc. 2011;8(2):30-1.

17. Xu L, Qin X, Sun W, Qiao J, Qiu Y, Zhu Z. Replication of Association Between 53 Single-Nucleotide Polymorphisms in a DNA-Based Diagnostic Test and AIS Progression in Chinese Han Population. Spine (Phila Pa 1976). 2016;41(4):306-10.

18. Tang QL, Julien C, Eveleigh R, Bourque G, Franco A, Labelle H, et al. A replication study for association of 53 single nucleotide polymorphisms in ScoliScore test with adolescent idiopathic scoliosis in French-Canadian population. Spine (Phila Pa 1976). 2015;40(8):537-43.

19. Burger EL, Perry J. ScoliScore: Is It Enough? Spine Deform. 2014;2(4):239-40.

20. Roye BD, Wright ML, Matsumoto H, Yorgova P, McCalla D, Hyman JE, et al. An Independent Evaluation of the Validity of a DNA-Based Prognostic Test for Adolescent Idiopathic Scoliosis. The Journal of bone and joint surgery American volume. 2015;97(24):1994-8.

21. Sharma S, Gao X, Londono D, Devroy SE, Mauldin KN, Frankel JT, et al. Genome-wide association studies of adolescent idiopathic scoliosis suggest candidate susceptibility genes. Hum Mol Genet. 2011;20(7):1456-66.

22. Takahashi Y, Kou I, Takahashi A, Johnson TA, Kono K, Kawakami N, et al. A genome-wide association study identifies common variants near LBX1 associated with adolescent idiopathic scoliosis. Nature genetics. 2011;43(12):1237-40.

23. Nelson LM, Chettier R, Ogilvie JW, Ward K. Candidate Genes for Susceptibility of Adolescent Idiopathic Scoliosis Identified Through a Large Genome-Wide Association Study. Scoliosis Research Society 46th Annual Meeting \& Course; Louisville, KY2011.

24. Kou I, Takahashi Y, Johnson TA, Takahashi A, Guo L, Dai J, et al. Genetic variants in GPR126 are associated with adolescent idiopathic scoliosis. Nature genetics. 2013;45(6):676-9.

25. Sharma S, Londono D, Eckalbar WL, Gao X, Zhang D, Mauldin K, et al. A PAX1 enhancer locus is associated with susceptibility to idiopathic scoliosis in females. Nature communications. 2015;6:6452.

26. Nada D, Julien C, Samuels ME, Moreau A. A Replication Study for Association of LBX1 Locus With Adolescent Idiopathic Scoliosis in French-Canadian Population. Spine (Phila Pa 1976). 2018;43(3):172-8.

27. Man GC, Tang NL, Chan TF, Lam TP, Li JW, Ng BK, et al. Replication Study for the Association of GWAS-associated Loci with Adolescent Idiopathic Scoliosis Susceptibility and Curve Progression in a Chinese Population. Spine (Phila Pa 1976). 2018.

28. Buchan JG, Alvarado DM, Haller GE, Cruchaga C, Harms MB, Zhang T, et al. Rare variants in FBN1 and FBN2 are associated with severe adolescent idiopathic scoliosis. Hum Mol Genet. 2014;23(19):5271-82.

29. Baschal EE, Wethey Cl, Swindle K, Baschal RM, Gowan K, Tang NL, et al. Exome sequencing identifies a rare HSPG2 variant associated with familial idiopathic scoliosis. G3. 2014;5(2):167-74. 
30. Patten SA, Margaritte-Jeannin P, Bernard JC, Alix E, Labalme A, Besson A, et al. Functional variants of POC5 identified in patients with idiopathic scoliosis. The Journal of clinical investigation. 2015;125(3):1124-8.

31. Grauers A, Wang J, Einarsdottir E, Simony A, Danielsson A, Akesson K, et al. Candidate gene analysis and exome sequencing confirm LBX1 as a susceptibility gene for idiopathic scoliosis. The spine journal : official journal of the North American Spine Society. 2015;15(10):2239-46.

32. Haller G, Alvarado D, McCall K, Yang P, Cruchaga C, Harms M, et al. A polygenic burden of rare variants across extracellular matrix genes among individuals with adolescent idiopathic scoliosis. Hum Mol Genet. 2016;25(1):202-9.

33. Gao W, Chen C, Zhou T, Yang S, Gao B, Zhou H, et al. Rare coding variants in MAPK7 predispose to adolescent idiopathic scoliosis. Hum Mutat. 2017;38(11):1500-10.

34. Baschal EE, Terhune EA, Wethey Cl, Baschal RM, Robinson KD, Cuevas MT, et al. Idiopathic Scoliosis Families Highlight Actin-Based and Microtubule-Based Cellular Projections and Extracellular Matrix in Disease Etiology. G3. 2018;8(8):2663-72.

35. Terhune EA, Cuevas MT, Monley AM, Wethey Cl, Chen X, Cattell MV, et al. Mutations in KIF7 implicated in idiopathic scoliosis in humans and axial curvatures in zebrafish. Hum Mutat. 2020.

36. Einarsdottir E, Grauers A, Wang J, Jiao H, Escher SA, Danielsson A, et al. CELSR2 is a candidate susceptibility gene in idiopathic scoliosis. PloS one. 2017;12(12):e0189591.

37. Cheng JC, Castelein RM, Chu WC, Danielsson AJ, Dobbs MB, Grivas TB, et al. Adolescent idiopathic scoliosis. Nat Rev Dis Primers. 2015;1:15030.

38. Dunwoodie SL, Kusumi K. The Genetics and Development of Scoliosis. Cham: Springer International Publishing : Imprint: Springer; 2018.

39. Grauers A, Einarsdottir E, Gerdhem P. Genetics and pathogenesis of idiopathic scoliosis. Scoliosis Spinal Disord. 2016;11:45.

40. Kouwenhoven JW, Castelein RM. The pathogenesis of adolescent idiopathic scoliosis: review of the literature. Spine (Phila Pa 1976). 2008;33(26):2898-908.

41. Alzu'bi AA, Zhou L, Watzlaf VJM. Genetic Variations and Precision Medicine. Perspect Health Inf Manag. 2019;16(Spring):1a.

42. Guyatt GH, Oxman AD, Vist GE, Kunz R, Falck-Ytter Y, Alonso-Coello P, et al. GRADE: an emerging consensus on rating quality of evidence and strength of recommendations. BMJ. 2008;336(7650):924-6.

43. Guyatt GH, Oxman AD, Kunz R, Falck-Ytter Y, Vist GE, Liberati A, et al. Going from evidence to recommendations. BMJ. 2008;336(7652):1049-51.

44. Guyatt GH, Oxman AD, Kunz R, Vist GE, Falck-Ytter Y, Schunemann HJ, et al. What is "quality of evidence" and why is it important to clinicians? BMJ. 2008;336(7651):995-8.

45. Sohani ZN, Meyre D, de Souza RJ, Joseph PG, Gandhi M, Dennis BB, et al. Assessing the quality of published genetic association studies in meta-analyses: the quality of genetic studies (Q-Genie) tool. 
BMC Genet. 2015;16:50.

46. Cochrane Handbook for Systematic Reviews of Interventions 2021 [updated February 2021. Available from: www.training.cochrane.org/handbook.

47. Page MJ, McKenzie JE, Bossuyt PM, Boutron I, Hoffmann TC, Mulrow CD, et al. The PRISMA 2020 statement: an updated guideline for reporting systematic reviews. BMJ. 2021;372:n71.

48. Moher D, Shamseer L, Clarke M, Ghersi D, Liberati A, Petticrew M, et al. Preferred reporting items for systematic review and meta-analysis protocols (PRISMA-P) 2015 statement. Syst Rev. 2015;4:1.

49. Shamseer L, Moher D, Clarke M, Ghersi D, Liberati A, Petticrew M, et al. Preferred reporting items for systematic review and meta-analysis protocols (PRISMA-P) 2015: elaboration and explanation. BMJ. 2015;350:g7647.

50. Terhune E. HMN, Heyn P, Piper C. . A Systematic Review of Genetic Variants Associated With Adolescent Idiopathic Scoliosis PROSPERO: National Institute for Health Research; 2021 [CRD42021243253]. Available from: https://www.crd.york.ac.uk/prospero/display_record.php? ID=CRD42021243253.

51. Harzing A. Publish or Perish 2007 [Available from: https://harzing.com/resources/publish-or-perish.

52. McGowan J, Sampson M, Salzwedel DM, Cogo E, Foerster V, Lefebvre C. PRESS Peer Review of Electronic Search Strategies: 2015 Guideline Statement. J Clin Epidemiol. 2016;75:40-6.

53. Bomba L, Walter K, Soranzo N. The impact of rare and low-frequency genetic variants in common disease. Genome Biol. 2017;18(1):77.

54. Cowell HR, Hall JN, MacEwen GD. Genetic aspects of idiopathic scoliosis. A Nicholas Andry Award essay, 1970. Clinical orthopaedics and related research. 1972;86:121-31.

55. Riseborough EJ, Wynne-Davies R. A genetic survey of idiopathic scoliosis in Boston, Massachusetts. The Journal of bone and joint surgery American volume. 1973;55(5):974-82.

56. Beals RK. Nosologic and genetic aspects of scoliosis. Clinical orthopaedics and related research. 1973(93):23-32.

57. Robin GC, Cohen T. Familial scoliosis. A clinical report. J Bone Joint Surg Br. 1975;57(2):146-8.

58. Lowe TG, Edgar M, Margulies JY, Miller NH, Raso VJ, Reinker KA, et al. Etiology of idiopathic scoliosis: current trends in research. The Journal of bone and joint surgery American volume. 2000;82-A(8):1157-68.

59. Ogilvie JW, Braun J, Argyle V, Nelson L, Meade M, Ward K. The search for idiopathic scoliosis genes. Spine (Phila Pa 1976). 2006;31(6):679-81.

60. Miller NH. Genetics of familial idiopathic scoliosis. Clinical orthopaedics and related research. 2007;462:6-10.

61. Fan YH, Song YQ, Chan D, Takahashi Y, Ikegawa S, Matsumoto M, et al. SNP rs11190870 near LBX1 is associated with adolescent idiopathic scoliosis in southern Chinese. J Hum Genet. 2012;57(4):244-6. 
62. Jiang H, Qiu X, Dai J, Yan H, Zhu Z, Qian B, et al. Association of rs11190870 near LBX1 with adolescent idiopathic scoliosis susceptibility in a Han Chinese population. European spine journal : official publication of the European Spine Society, the European Spinal Deformity Society, and the European Section of the Cervical Spine Research Society. 2013;22(2):282-6.

63. Gao W, Peng Y, Liang G, Liang A, Ye W, Zhang L, et al. Association between common variants near LBX1 and adolescent idiopathic scoliosis replicated in the Chinese Han population. PloS one. 2013;8(1):e53234.

64. Londono D, Kou I, Johnson TA, Sharma S, Ogura Y, Tsunoda T, et al. A meta-analysis identifies adolescent idiopathic scoliosis association with LBX1 locus in multiple ethnic groups. J Med Genet. 2014;51(6):401-6.

65. Chen S, Zhao L, Roffey DM, Phan P, Wai EK. Association of rs11190870 near LBX1 with adolescent idiopathic scoliosis in East Asians: a systematic review and meta-analysis. The spine journal : official journal of the North American Spine Society. 2014;14(12):2968-75.

66. Liang J, Xing D, Li Z, Chua S, Li S. Association Between rs11190870 Polymorphism Near LBX1 and Susceptibility to Adolescent Idiopathic Scoliosis in East Asian Population: A Genetic Meta-Analysis. Spine (Phila Pa 1976). 2014;39(11):862-9.

67. Zhu Z, Tang NL, Xu L, Qin X, Mao S, Song Y, et al. Genome-wide association study identifies new susceptibility loci for adolescent idiopathic scoliosis in Chinese girls. Nature communications. 2015;6:8355.

68. Chettier R, Nelson L, Ogilvie JW, Albertsen HM, Ward K. Haplotypes at LBX1 have distinct inheritance patterns with opposite effects in adolescent idiopathic scoliosis. PloS one. 2015;10(2):e0117708.

69. Cao Y, Min J, Zhang Q, Li H, Li H. Associations of LBX1 gene and adolescent idiopathic scoliosis susceptibility: a meta-analysis based on 34,626 subjects. BMC musculoskeletal disorders. 2016;17:309.

70. Liu S, Wu N, Zuo Y, Zhou Y, Liu J, Liu Z, et al. Genetic Polymorphism of LBX1 Is Associated With Adolescent Idiopathic Scoliosis in Northern Chinese Han Population. Spine (Phila Pa 1976). 2017;42(15):1125-9.

71. Masselink W, Masaki M, Sieiro D, Marcelle C, Currie PD. Phosphorylation of Lbx1 controls lateral myoblast migration into the limb. Developmental biology. 2017;430(2):302-9.

72. Li YL, Gao SJ, Xu H, Liu Y, Li HL, Chen XY, et al. The association of rs11190870 near LBX1 with the susceptibility and severity of AIS, a meta-analysis. Int J Surg. 2018;54(Pt A):193-200.

73. Xu L, Wu Z, Xia C, Tang N, Cheng JCY, Qiu Y, et al. A Genetic Predictive Model Estimating the Risk of Developing Adolescent Idiopathic Scoliosis. Curr Genomics. 2019;20(4):246-51.

74. Xu JF, Yang GH, Pan XH, Zhang SJ, Zhao C, Qiu BS, et al. Association of GPR126 gene polymorphism with adolescent idiopathic scoliosis in Chinese populations. Genomics. 2015;105(2):101-7.

75. Qin X, Xu L, Xia C, Zhu W, Sun W, Liu Z, et al. Genetic Variant of GPR126 Gene is Functionally Associated With Adolescent Idiopathic Scoliosis in Chinese Population. Spine (Phila Pa 1976). 2017;42(19):E1098-E103. 
76. Kou I, Watanabe K, Takahashi Y, Momozawa Y, Khanshour A, Grauers A, et al. A multi-ethnic metaanalysis confirms the association of rs6570507 with adolescent idiopathic scoliosis. Sci Rep. 2018;8(1):11575.

77. Liu G, Liu S, Lin M, Li X, Chen W, Zuo Y, et al. Genetic polymorphisms of GPR126 are functionally associated with PUMC classifications of adolescent idiopathic scoliosis in a Northern Han population. J Cell Mol Med. 2018;22(3):1964-71.

78. Khanshour AM, Wise CA. The Genetic Architecture of Adolescent Idiopathic Scoliosis. In: Machida M, Weinstein SL, Dubousset J, editors. Pathogenesis of Idiopathic Scoliosis. Tokyo: Springer Japan; 2018. p. 51-74.

79. Hadley-Miller N, Mims B, Milewicz DM. The potential role of the elastic fiber system in adolescent idiopathic scoliosis. The Journal of bone and joint surgery American volume. 1994;76(8):1193-206.

80. Miller NH, Mims B, Child A, Milewicz DM, Sponseller P, Blanton SH. Genetic analysis of structural elastic fiber and collagen genes in familial adolescent idiopathic scoliosis. $J$ Orthop Res. 1996;14(6):994-9.

81. Wise CA, Sepich D, Ushiki A, Khanshour AM, Kidane YH, Makki N, et al. The cartilage matrisome in adolescent idiopathic scoliosis. Bone Res. 2020;8(1):13.

82. Maqsood A, Frome DK, Gibly RF, Larson JE, Patel NM, Sarwark JF. IS (Idiopathic Scoliosis) etiology: Multifactorial genetic research continues. A systematic review 1950 to 2017. J Orthop. 2020;21:4216.

83. Jiang H, Yang Q, Liu Y, Guan Y, Zhan X, Xiao Z, et al. Association between ladybird homeobox 1 gene polymorphisms and adolescent idiopathic scoliosis: A MOOSE-compliant meta-analysis. Medicine (Baltimore). 2019;98(27):e16314.

84. Chen S, Zhao L, Roffey DM, Phan P, Wai EK. Association between the ESR1 -351A>G single nucleotide polymorphism (rs9340799) and adolescent idiopathic scoliosis: a systematic review and meta-analysis. European spine journal : official publication of the European Spine Society, the European Spinal Deformity Society, and the European Section of the Cervical Spine Research Society. 2014;23(12):2586-93.

85. Zhao L, Roffey DM, Chen S. Association Between the Estrogen Receptor Beta (ESR2) Rs1256120 Single Nucleotide Polymorphism and Adolescent Idiopathic Scoliosis: A Systematic Review and Meta-Analysis. Spine (Phila Pa 1976). 2017;42(11):871-8.

86. Sobhan MR, Mahdinezhad-Yazdi M, Dastgheib SA, Jafari M, Raee-Ezzabadi A, Neamatzadeh H. Association of ESRalpha Xbal A > G, ESRalpha Pvull T $>$ C and ESRbeta AlwNI T $>$ C Polymorphisms with the Risk of Developing Adolescent Idiopathic Scoliosis: A Systematic Review and Genetic Metaanalysis. Rev Bras Ortop (Sao Paulo). 2020;55(1):8-16.

87. Yin X, Wang H, Guo J, Zhang L, Zhang Y, Li L, et al. Association of vitamin D receptor Bsml rs1544410 and Apal rs7975232 polymorphisms with susceptibility to adolescent idiopathic scoliosis: A systematic review and meta-analysis. Medicine (Baltimore). 2018;97(2):e9627. 
88. Dai J, Lv ZT, Huang JM, Cheng P, Fang H, Chen AM. Association between polymorphisms in vitamin D receptor gene and adolescent idiopathic scoliosis: a meta-analysis. European spine journal : official publication of the European Spine Society, the European Spinal Deformity Society, and the European Section of the Cervical Spine Research Society. 2018;27(9):2175-83.

89. Harris PA, Taylor R, Thielke R, Payne J, Gonzalez N, Conde JG. Research electronic data capture (REDCap)-a metadata-driven methodology and workflow process for providing translational research informatics support. J Biomed Inform. 2009;42(2):377-81.

\section{Figures}
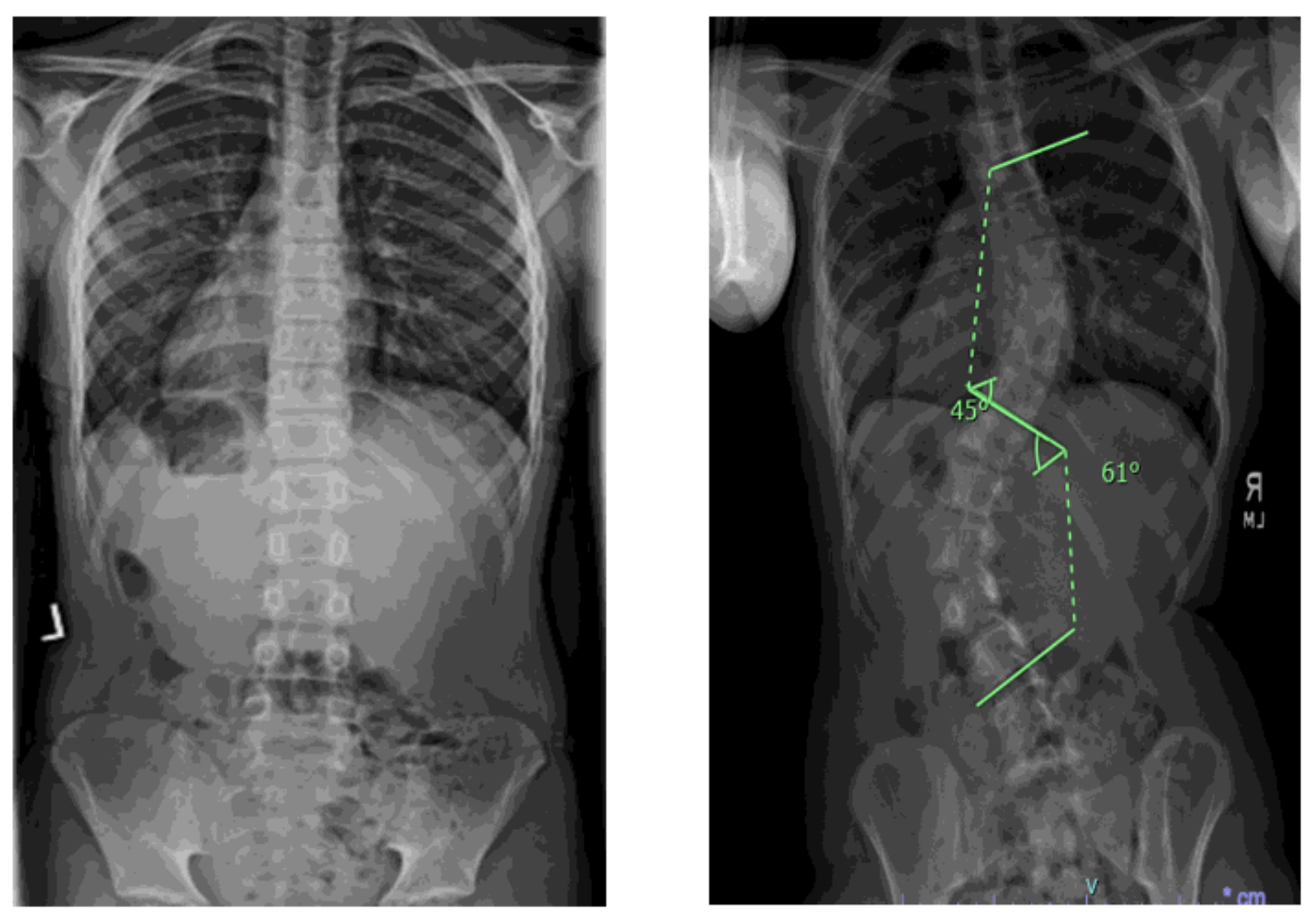

\section{Figure 1}

Radiographs of children with a normal spine (left) and severe adolescent idiopathic scoliosis (AIS) (right), shown with Cobb angle measurements. 


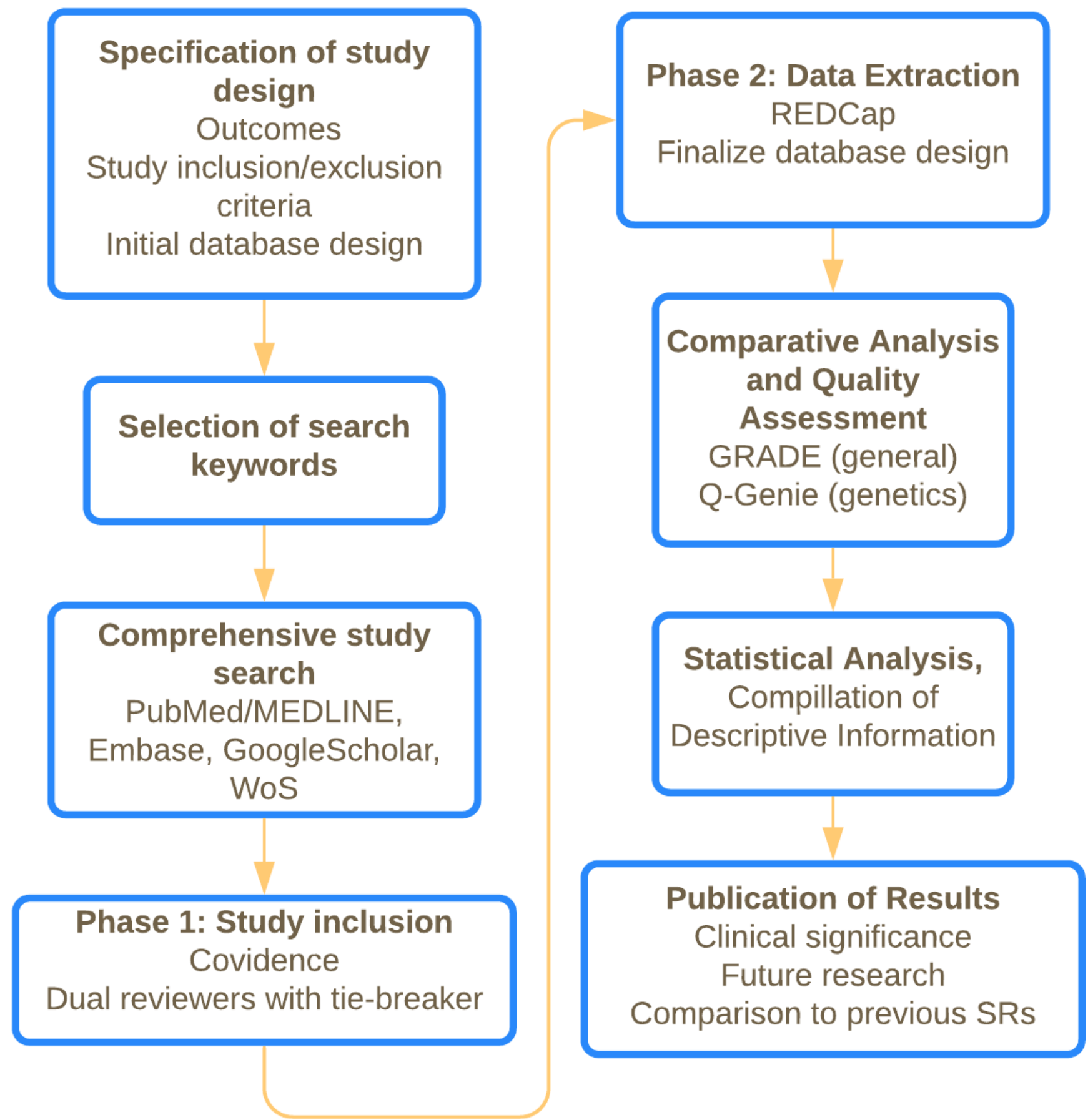

Figure 2

Flow chart overview of study design.

\section{Supplementary Files}

This is a list of supplementary files associated with this preprint. Click to download. 
- SupplementalFile1PRISMA.docx

- SupplementalFile2SearchStrategies.docx

- SupplementalFile3InclusionExclusion.docx 